\title{
Synergy between SIRT1 and SIRT6 helps recognize DNA breaks and potentiate the DNA damage response and repair
}

Fanbiao Meng, ${ }^{1,2,8}$ Minxian Qian, ${ }^{1,3,8}$ Bin Peng, ${ }^{1,8}$ Xiaohui Wang, ${ }^{1,5}$ Linyuan Peng, ${ }^{1,3}$ Kang Zheng, ${ }^{6}$ Zuojun Liu, ${ }^{1,3}$ Xiaolong Tang, ${ }^{1,3}$ Shuju Zhang, ${ }^{1}$ Shimin Sun, ${ }^{1,6}$ Xinyue Cao, ${ }^{1,3}$ Qiuxiang Pang, ${ }^{6}$ Bosheng Zhao, ${ }^{6}$ Wenbin Ma, ${ }^{7}$ Zhou Songyang, ${ }^{7} \mathrm{Bo} \mathrm{Xu},{ }^{2}$ Wei-Guo Zhu, ${ }^{1,5}$ Xingzhi Xu, ${ }^{1,5^{*}}$ Baohua Liu ${ }^{1,3,4,5^{*}}$

${ }^{1}$ Guangdong Key Laboratory of Genome Stability and Human Disease Prevention, Department of Biochemistry \& Molecular Biology, School of Basic Medical Sciences, Shenzhen University Health Science Center, Shenzhen 518055, China

${ }^{2}$ The Key Laboratory of Breast Cancer Prevention and Therapy, Ministry of Education, Tianjin's Clinical Research Center for Cancer, Tianjin Medical University Cancer Institute and Hospital, National Clinical Research Center for Cancer, Tianjin 300006, China

${ }^{3}$ Shenzhen Key Laboratory for Systemic Aging and Intervention, National Engineering Research Center for Biotechnology (Shenzhen), Shenzhen University Health Science Center, Shenzhen 518055, China

${ }^{4}$ Guangdong Provincial Key Laboratory of Regional Immunity and Diseases, School of Basic Medical Sciences, Shenzhen University Health Science Center, Shenzhen 518055, China

${ }^{5}$ International Cancer Center, Shenzhen University Health Science Center, Shenzhen 518055, China

${ }^{6}$ Anti-aging \& Regenerative Medicine Research Institution, School of Life Sciences, Shandong University of Technology, Zibo 255049, China

${ }^{7}$ Key Laboratory of Gene Engineering of the Ministry of Education and State Key

Laboratory for Biocontrol, School of Life Sciences, Sun Yat-sen University, Guangzhou, China

${ }^{8}$ These authors contribute equally to this work.

* Correspondence should be addressed to Dr Baohua Liu (ppliew@szu.edu.cn) and Dr Xingzhi Xu (xingzhi.xu@szu.edu.cn). 


\section{Summary}

The DNA damage response (DDR) is a highly orchestrated process but how double-strand DNA breaks (DSBs) are initially recognized is unclear. Here, we show that polymerized SIRT6 deacetylase recognizes DSBs and potentiates the DDR. First, SIRT1 deacetylates SIRT6 at residue K33, which is important for SIRT6 polymerization and mobilization toward DSBs. Then, K33-deacetylated SIRT6 anchors to $\gamma \mathrm{H} 2 \mathrm{AX}$, allowing its retention on and subsequent remodeling of local chromatin. We show that a K33R mutation that mimics hypoacetylated SIRT6 can rescue defective DNA repair as a result of SIRT1 deficiency in cultured cells. These data highlight the synergistic action between SIRTs in the spatiotemporal regulation of the DDR and DNA repair.

Key Words: SIRT1, SIRT6, DNA damage response (DDR), double strand DNA breaks (DSB), deacetylation, $\gamma \mathrm{H} 2 \mathrm{AX}$. 


\section{Introduction}

DNA damage can be induced by various endogenous and exogenous agents. Upon detection of damage, the DNA damage response (DDR) is immediately elicited to regain genomic integrity via chromatin remodeling, signaling transduction and amplification (1). Double-strand breaks (DSBs) are the most severe type of DNA lesion; they are recognized by the Mre11-Rad50-Nbs1 (MRN) complex, which recruits and activates phosphatidylinositol 3-kinase-like protein kinase ataxia-telangiectasia mutated (ATM) or ATM- and Rad3-related (ATR). H2AX is then rapidly phosphorylated $(\gamma \mathrm{H} 2 \mathrm{AX})$ by $\mathrm{ATM} / \mathrm{ATR}$, and serves as a platform to localize repair proteins near to the DNA breaks (2). Simultaneously, various histone-modifying enzymes, heterochromatin factors and ATP-dependent chromatin remodelers work cooperatively to relax the chromatin structure and ensure that additional repair factors have access to the DSBs (3). Despite all these advances in understanding the DDR, how DSBs are initially and precisely recognized is largely unknown.

$\mathrm{NAD}^{+}$-dependent sirtuins belong to class III histone deacetylases (HDACs) (4). Seven sirtuins (SIRT1-7) with various enzymatic activities and physiological functions are expressed in mammals. SIRT1, 6 and 7 localize in the nucleus and seem to be most relevant to the DDR as their depletion causes growth retardation, a defective DDR and DNA repair and premature aging (5-7). Upon DNA damage, SIRT1 redistributes on chromatin, co-localizes with $\gamma \mathrm{H} 2 \mathrm{AX}$, and deacetylates XPA, NBS1 and Ku70 to regulate nucleotide excision repair, homologous recombination (HR) and non-homologous end-joining (NHEJ) (8-11). Depleting Sirt1 in mouse fibroblasts impairs the DDR and leads to genomic instability (6). SIRT6 is one of the earliest factors recruited to DSBs; it initiates the subsequent recruitment of SNF2H, H2AX, DNA-PKcs and PARP1 (12-15). SIRT6 mono-ribosylates PARP1 to enhance its activity (16). Despite their rapid mobilization to DNA breaks, the triggers for sirtuin recruitment are obscure $(7,17,18)$.

Here, we aimed to delineate the mechanisms underlying DSB recognition. We found that SIRT6 polymerizes and directly recognizes DSBs via a putative DNA-binding pocket consisting of $\mathrm{N}$ - and $\mathrm{C}$-termini from two adjacent molecules. SIRT1 interacts 
with SIRT6 and deacetylates it at K33, thus allowing its polymerization and recognition of DSBs. A K33R mutant, mimicking hypoacetylated SIRT6, could rescue DNA repair defects in SIRT1 knockout (KO) cells. Our data highlight an essential synergy between SIRT1 and SIRT6 in the spatiotemporal regulation of the DDR.

\section{Results}

\section{SIRT6 directly recognizes DNA double-strand breaks}

Nuclear SIRTs (SIRT1/6/7) are quickly mobilized to DSBs (Figure S1) and serve as a scaffold for DNA repair factors $(7,17,18)$. Intriguingly, these nuclear SIRTs are also activated by RNA and the nucleosome $(19,20)$. We thus reasoned that SIRTs might directly sense DNA breaks, especially DSBs. To test our hypothesis, we established a molecular docking simulation using AutoDock Vina software (21). We obtained crystal structures for SIRT1 (PDB code 4I5I) (22), SIRT6 (PDB code 3PKI) (23) and SIRT7 (PDB code 5IQZ) (24) from the Protein Data Bank (https://www.rcsb.org). We removed the heteroatoms and added Gasteiger charges to the atoms. A DSB structure was extracted from the PDB code 4DQY (25). As SIRTs use $\mathrm{NAD}^{+}$as a co-substrate in amide bond hydrolysis, which shares a similar skeleton to the phosphate, base and ribose groups on broken DSB ends, we included $\mathrm{NAD}^{+}$as a simulation control.

We found that the binding affinity between $\mathrm{NAD}^{+}$and all nuclear SIRTs was within the range of -8 to $-10 \mathrm{kcal} / \mathrm{mol}$ (Figure $1 \mathrm{~A}$ ). Surprisingly, only the binding between the DSB and SIRT6 was energetically favored $(-12.7 \mathrm{kcal} / \mathrm{mol})$, being even lower than that of $\mathrm{NAD}^{+}$(Figures 1A,B). This finding suggested that SIRT6 might directly bind to DSBs and prompted us to gain further experimental evidence.

We next generated a DSB-mimicking biotin-conjugated DNA duplex and performed an in vitro pulldown assay. Recombinant SIRT6 (rSIRT6), but not rSIRT1 or rSIRT7, bound to the DNA duplex (Figure 1C). This finding was verified by a fluorescence polarization (FP) assay using a FAM-labeled DNA duplex. Here, we observed dynamic FP $(\mathrm{Kd}=166.3 \mathrm{nM}$; Figure 1D), supporting a specific and direct interaction between the DNA duplex and rSIRT6. By contrast, the FP was minimal for both rSIRT1 and rSIRT7 (Figure 1D). To interrogate whether such binding is specific to broken DNA, we repeated the pulldown assay in the presence of unlabeled linear or circular DNA. While linearized DNA inhibited rSIRT6 binding to the DNA duplex, circular DNA had a minimal effect (Figure 1E). Together, these data indicate that 
SIRT6, but not SIRT1 or SIRT7 recognizes and directly binds to DSBs.

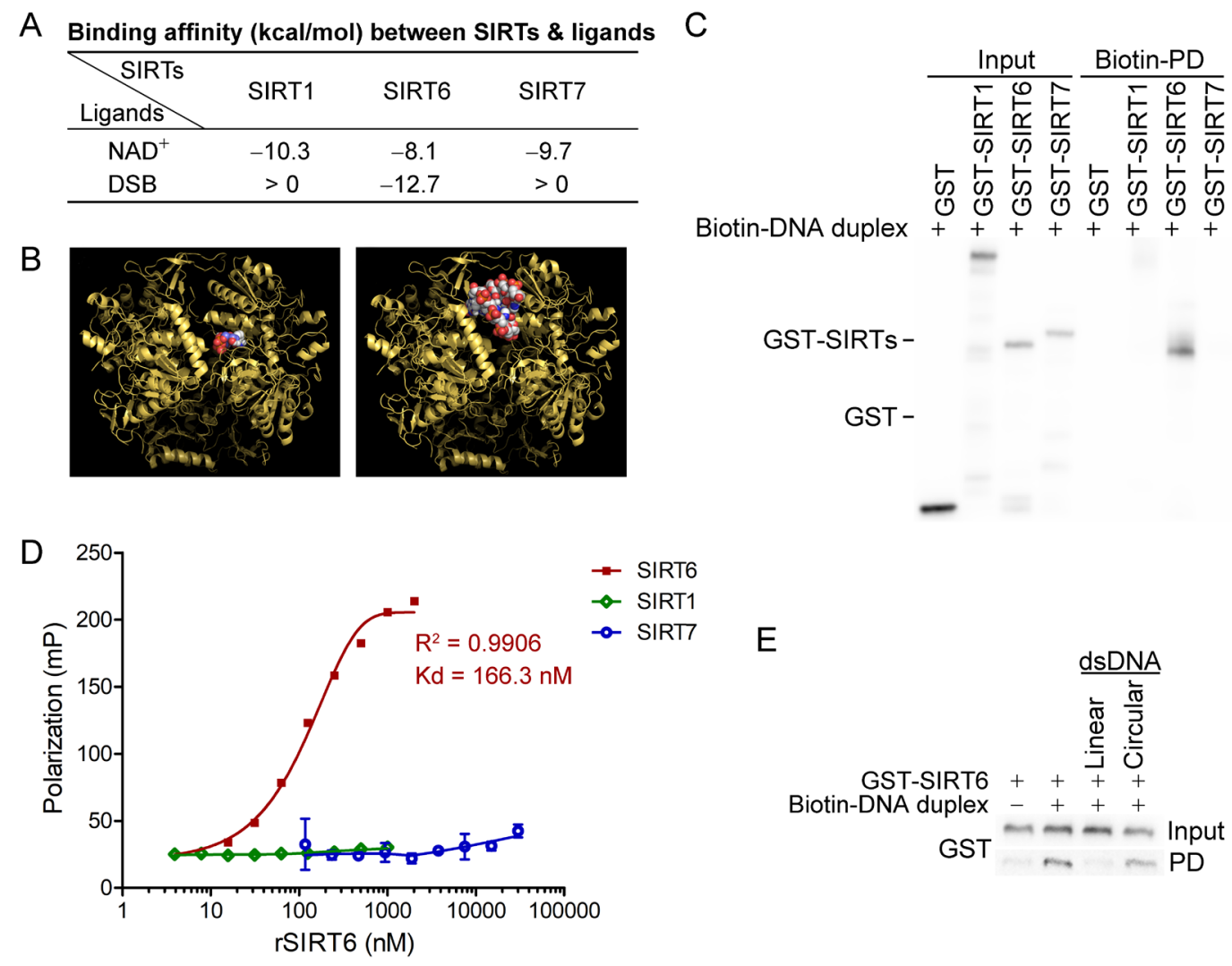

Figure 1. SIRT6 directly recognizes DNA breaks.

(A) The predicted binding affinity ( $\mathrm{kcal} / \mathrm{mol})$ between sirtuins (SIRTs) and ligands $\left(\mathrm{NAD}^{+}\right.$and a DSB).

(B) Molecular docking of SIRT6 with a DSB (right) and NAD ${ }^{+}$(left).

(C) A biotin-labeled DNA duplex was incubated with the indicated recombinant SIRTs. Streptavidin beads were pulled down and blotted with anti-GST and anti-SIRT1 antibodies.

(D) The fluorescence polarization (FP) of FAM-labeled DNA was detected after incubation with GST-SIRT1, GST-SIRT6 or GST-SIRT7.

(E) A pulldown assay comprising a biotin-labeled DNA duplex with GST-SIRT6 in the presence of unlabeled linear DNA or circular DNA.

\section{SIRT6 is dynamically acetylated in the $\mathrm{N}$ terminus at $\mathrm{K33}$}

As predicted from the crystallographic data, SIRT6s form an asymmetric hexamer (23) that generates three potential DSB binding pockets; each of these pockets consist of 
two N-termini and two C-termini from two adjacent molecules (Figure S2A). Both the N-termini and C-termini are essential for SIRT6 to associate with chromatin (26). To gain biochemical evidence for SIRT6 polymerization, we used a biomolecule fluorescence compensation system (BiFC). In brief, we cloned SIRT6 cDNA into either the N-terminal or C-terminal of a yellow fluorescence protein (YFP), herein termed N-SIRT6 and C-SIRT6. The yellow fluorescence was detectable by FACS only when N-SIRT6 directly interacted with C-SIRT6. After co-transfecting these constructs into HEK293 cells, we detected a strong fluorescence signal by FACS in $>24 \%$ cells (Figure S2B), suggesting a direct interaction between the two SIRT6 molecules. To confirm this SIRT6 polymerization event, we performed a co-immunoprecipitation (Co-IP) assay in HEK293 cells in which we had co-overexpressed FLAG-SIRT6 and HA-SIRT6. Consistently, we detected FLAG-SIRT6 in the anti-HA-SIRT6 immunoprecipitates (Figure S2C).

The DSB phosphate backbone is negatively charged. The positive-charged environment of SIRT6 thus favors its binding to DSBs. Indeed, one of our predicted DSB-binding pockets formed by two adjacent molecules in SIRT6 hexamer consisted of six positively charged residues at the edge, namely four arginine (R32/39) and two lysine (K33) residues (Figure S2D). Acetylation is the most redundant post-translational modification that converts positively charged $\mathrm{K}$ to neutral Kac. This property is utilized by proteins with a lysine-rich domain (KRD), e.g. Histones, Ku70 and $\mathrm{p} 53$, for mediating dynamic interactions with proteins harboring an acidic domain like SET (27). The heterodimerized Ku70 and Ku80 complex directly senses DSBs via a flexible C-termini containing multiple $\mathrm{K}$ residues, and regulates NHEJ (28). We therefore examined whether SIRT6 is (de)acetylated on these K residues thus sensing DSBs. We immunoprecipitated FLAG-SIRT6 with an anti-FLAG antibody and then probed the immunoprecipitate with anti-Kac antibodies. As shown, Kac was detected in the precipitated FLAG-SIRT6 immunocomplex (Figure 2A). We then purified FLAG-SIRT6 and performed high-resolution LC-MS/MS to identify which $\mathrm{K}$ residues are acetylated (Table S1). We identified K15 and K33 in the N-terminus. To confirm these acetylated $\mathrm{K}$ residues, we generated $\mathrm{K} 15 \mathrm{R}$ and $\mathrm{K} 33 \mathrm{R}$ point mutants, with K17R serving as a negative control. While neither K15R nor K17R affected the FLAG-SIRT6 acetylation level, K33R significantly inhibited it (Figures 2A and S3), supporting that K33 is dynamically (de)acetylated. 


\section{Dynamic SIRT6 K33 (de)acetylation regulates DSB sensing}

To understand the function of SIRT6 K33 acetylation, we examined whether it is required for binding to DSBs. We used our K33R mutant and generated a new K33Q mutant to mimic deacetylated or acetylated SIRT6, respectively (29). We also mutated SIRT6 H133 to a tyrosine residue (H133Y) to blunt SIRT6 enzymatic activity (18). $\mathrm{K} 33 \mathrm{Q}$ and H133Y binding to the DNA duplex was significantly compromised compared to WT and K33R binding (Figure 2B). Consistently, we recorded notable FP for SIRT6 K33R (Kd=104.9 nM) but hardly any FP for SIRT6 K33Q (Figure 2C).

We then monitored GFP-SIRT6 mobility in cells upon receipt of DNA damage. H133 is critical for enriching SIRT6 on chromatin (26). We reconstituted GPF-SIRT6 WT, K33Q, K33R and H133Y in Sirt6 ${ }^{-1}$ cells and monitored their recruitment to DSBs. While the K33Q and H133Y mutations significantly jeopardized efficient SIRT6 recruitment to DNA breaks, the SIRT6 K33R mutant retained such ability (Figures 2D-E). To gain more experimental support, we made use of an inducible DR-GFP reporter system that contains a unique I-SceI cutting site. In presence of triamcinolone acetonide (TA), the I-SceI-GR enzyme translocates to the nucleus within $10 \mathrm{~min}$ and generates DSBs, as evidenced by an increase in the $\gamma \mathrm{H} 2 \mathrm{AX}$ level (Figure 2F-G). We thus monitored the occupancy of SIRT6 on chromatin surrounding these induced DSBs, by chromatin immunoprecipitation (ChIP) and quantitative PCR, as previously described (30). Both the K33Q and H133Y mutations compromised SIRT6 recruitment to the sites of damage, whereas SIRT6 K33R recruitment was comparable to that of SIRT6 WT (Figures 2H).

Upon DNA damage, the acetylation levels of H3K9 and H3K56 decline, and after repair, goes back to the original level (31). H3K9ac and $\mathrm{H} 3 \mathrm{~K} 56 \mathrm{ac}$ are deacetylating targets of SIRT6, indicating that SIRT6 might contribute to the reduced H3K9ac and H3K56ac levels on the DSB-surrounding chromatin. Indeed, reconstituted SIRT6 WT and K33R downregulated the levels of H3K9ac and H3K56ac in Sirt6 ${ }^{-/}$cells, while K33Q and H133Y failed (Figures 2I and S4A). Further, the K33Q and H133Y mutations also affected SNF2H recruitment to DSBs (Figure S4B), which requires SIRT6 (18), but no effect was observed in the presence of the K33R mutation. Of note, neither K33R nor K33Q affected the deacetylase activity of SIRT6 (Figure S4C).

We next analyzed whether dynamic K33 (de)acetylation modulates SIRT6 
polymerization. We co-overexpressed HA-SIRT6 and various FLAG-SIRT6 mutants and performed a Co-IP assay. We detected FLAG-SIRT6 in the anti-HA immunoprecipitates, supporting that SIRT6 polymerization occurs (Figure S2C). While HA-SIRT6 bound to SIRT6 K33R to a similar extent as to SIRT6 WT, its binding to SIRT6 K33Q was significantly jeopardized. Of note, the enzyme-dead H133Y mutation also jeopardized SIRT6 polymerization. This finding is consistent with the fact that the H133 site is important for both SIRT6 deacetylase activity and for mediating the chromatin association (26). We confirmed this jeopardized polymerization in the $\mathrm{K} 33 \mathrm{Q}$ mutant condition by BiFC assay (Figure S5A,B). Together, these data implicate that dynamic SIRT6 K33 (de)acetylation modulates SIRT6 polymerization and thus DSB binding.| 
Figure 2

A
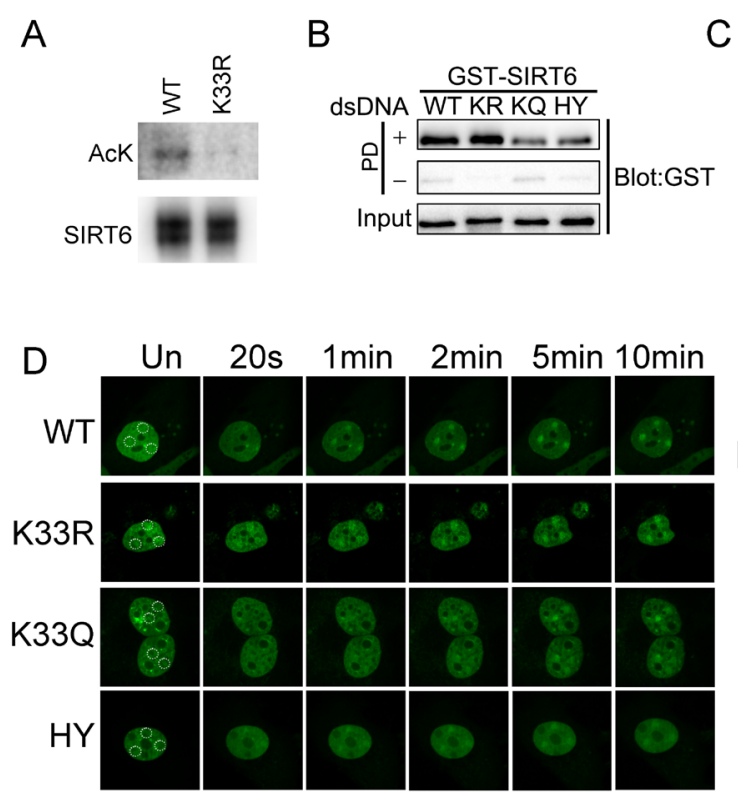

$\mathrm{F}$

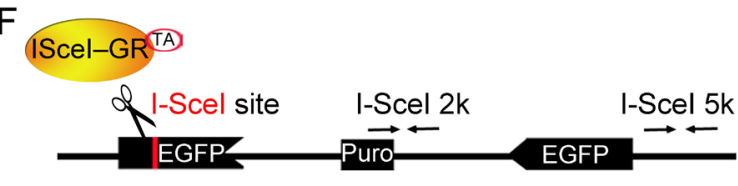

G

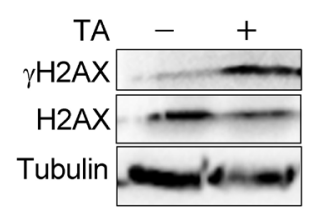

I

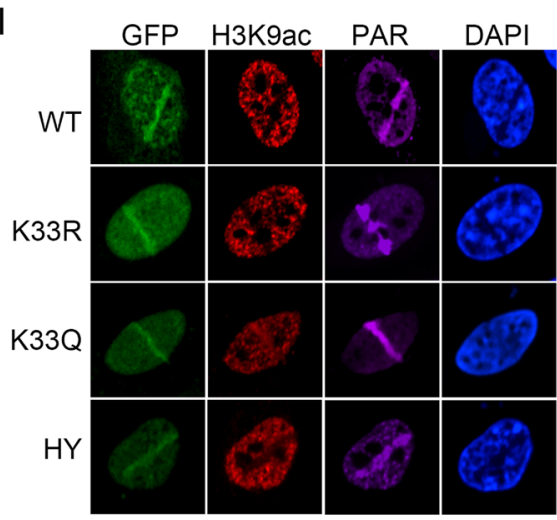

C

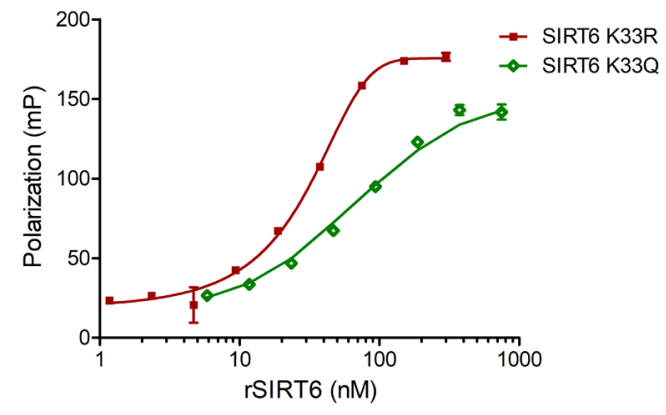

$E$

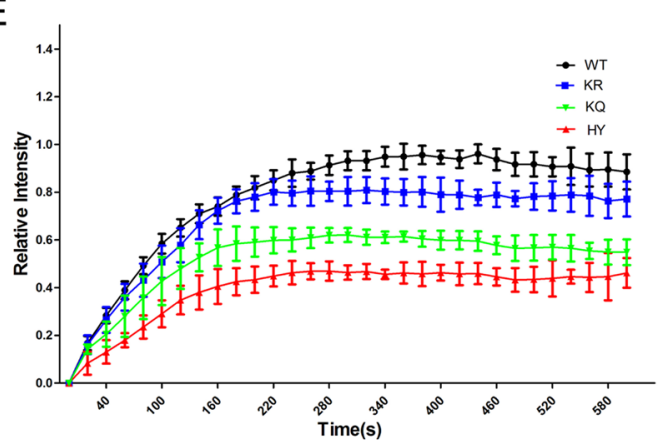

$\mathrm{H}$

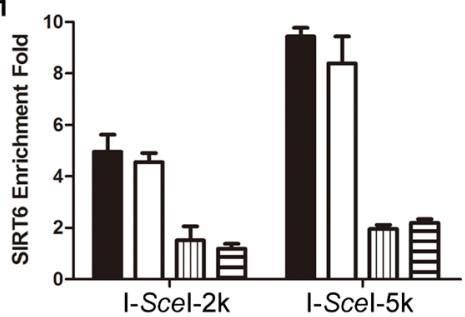

Figure 2. SIRT6 K33 (de)acetylation regulates DSB binding

(A) The acetylation levels of WT and K33R mutated SIRT6 were assessed by western blotting.

(B) A pulldown assay showing the interactions between a biotin-labeled DNA duplex and the indicated GST-SIRT6 peptides. 
(C) Fluorescence polarization (FP) of Fam labeled DNA was detected after incubating with GST-SIRT6 K133R or K133Q.

(D-E) The dynamic recruitment of GFP-SIRT6, K33R, K33Q and HY (H133Y) to laser-induced DNA breaks was assessed by confocal microscopy. Representative images are shown (D) and the white dot circles indicate the damage sites. The relative intensity was calculated in Fiji (Image $\mathbf{J}^{\circledR}$ ) (E).

(F) A schematic of the DR-GFP construct, which contains a single I-SceI site to create DNA break in the presence of triamcinolone acetonide and I-SceI endonuclease. The positions of the amplification primers $2 \mathrm{~K}$ and $5 \mathrm{~K}$ downstream I-SceI site used for q-PCR are indicated.

(G) DNA breaks were generated in DR-GFP stably transfected Hela cells after triamcinolone acetonide (TA) treatment for $20 \mathrm{~min}$, as evidenced by elevated $\gamma \mathrm{H} 2 \mathrm{AX}$ staining.

(H) ChIP-PCR analysis of the enrichment of SIRT6 and various SIRT6 mutants at DNA break sites. The relative SIRT6 expression was confirmed by western blotting. The qPCR data were normalized to the input DNA and a sample not treated with I-SceI endonuclease (no cut). ${ }^{*} p<0.05$.

(I) Fluorescence imaging of GFP-SIRT6 WT, indicated mutants, immune-stained H3K9ac and H3K56ac in Sirt6 ${ }^{-1-}$ MEFs. PAR immunostaining reveals the damage site.

\section{SIRT6 interacts with SIRT1}

Having confirmed SIRT6 (de)acetylation, we moved to examine potential deacetylase of SIRT6. To this end, we first tested the effect of various HDAC inhibitors on SIRT6 acetylation level. We noticed that the level of acetylated SIRT6 was largely elevated in the presence of the class III HDAC (SIRTs) inhibitor nicotinamide (NAM) or the SIRT1-specific inhibitor Ex527, but not the class I/II HADC inhibitor Trichostatin A (TSA) (Figure S6). This finding suggested that SIRT1 might be involved in SIRT6 deacetylation. Indeed, co-IP and western blotting revealed that FLAG-SIRT6 interacted with endogenous SIRT1 (Figure 3A) and vice versa in HEK293 cells (Figure 3B). In addition, we detected SIRT1 in the anti-SIRT6 immunoprecipitates and vice versa (Figure 3C,D). A GST pulldown assay confirmed that His-SIRT1 directly interacted with GST-SIRT6 (Figure 3E). Further, we observed co-localization 
between SIRT6 and SIRT1 by confocal microscopy in cells co-transfected with GFP-SIRT6 and DsRed-SIRT1 or in cells co-stained with specific antibodies (Figures $3 \mathrm{~F}$ and $\mathrm{S} 7 \mathrm{~A})$.

SIRTs contain a conserved Sir2 domain and flexible N-termini and C-termini. To locate the exact SIRT6 domains that interact with SIRT1, we deleted the N-terminus and C-terminus, as previously reported (26) (Figure S7B,C). Western blotting analysis showed that the interaction between SIRT6 and SIRT1 was lost if the N-terminus or C-terminus of SIRT6 was deleted (Figure 3G). As the C-terminus contains the nuclear location signal (26), we speculate that its depletion may restrict SIRT6 in cytoplasm, thus preventing the interaction between SIRT1 and SIRT6. Thus, the data indicate that SIRT6 physically interacts with SIRT1, most likely through the N-terminus of SIRT6.

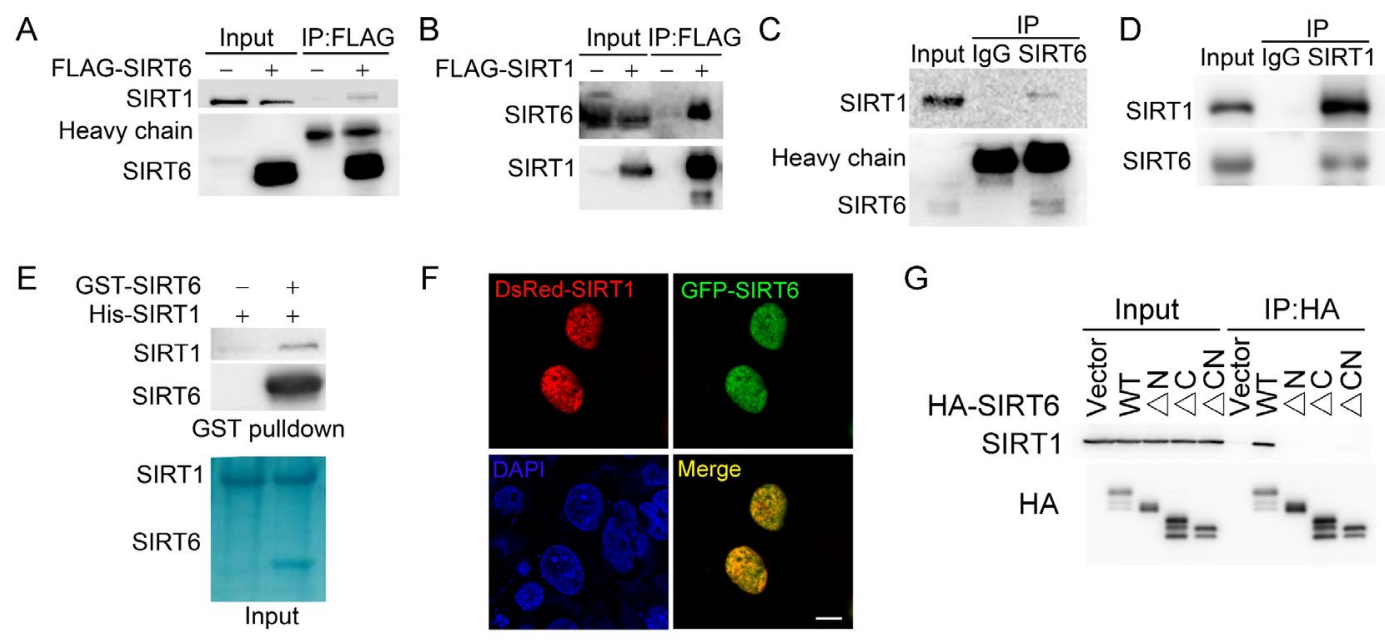

Figure 3. SIRT6 interacts with SIRT1.

(A) Western blot analysis of SIRT1 levels in anti-FLAG immunoprecipitates in HEK293 cells transfected with FLAG-SIRT6 or an empty vector.

(B) Western blot analysis of SIRT6 levels in anti-FLAG immunoprecipitates in HEK293 cells transfected with FLAG-SIRT1 or an empty vector.

(C) Western blot analysis of SIRT1 in anti-SIRT6 immunoprecipitates in Hela cells.

(D) Western blot analysis of SIRT6 in anti-SIRT1 immunoprecipitates in Hela cells.

(E) GST pulldown assay between GST-SIRT6 and His-SIRT1 in vitro.

(F) Representative images of DsRed-SIRT1 and GFP-SIRT6 localization in U2OS cells, determined by confocal microscopy. Scale bar, $10 \mu \mathrm{m}$.

(G) Co-immunoprecipitation and western blot analysis of the interactions between FLAG-SIRT1 and HA-SIRT6 $\Delta \mathrm{N}$ (N-terminus deleted), $\Delta \mathrm{C}$ (C-terminus deleted) and 
$\Delta \mathrm{CN}(\mathrm{N}-/ \mathrm{C}$-termini deleted) in HEK293 cells.

\section{SIRT1 deacetylates SIRT6 at K33}

We next examined whether SIRT1 deacetylates SIRT6 via the direct interaction that we identified above. Overexpression of SIRT1 but not of other sirtuins inhibited FLAG-SIRT6 acetylation (Figure 4A). Conversely, knocking down SIRT1 significantly upregulated endogenous SIRT6 acetylation in HEK293 cells (Figure 4B). Furthermore, the SIRT6 acetylation level decreased in the presence of ectopic SIRT1 but not in the presence of its catalytic mutant SIRT1-H363Y (Figure 4C), despite the two proteins still showing a physical interaction, suggesting that SIRT6 is likely a deacetylation target of SIRT1. To test our hypothesis, we established an in vitro deacetylation assay. We eluted recombinant FLAG-SIRT6 with a FLAG peptide from HEK293 cell lysate. We found that SIRT1 deacetylated SIRT6 in the presence of $\mathrm{NAD}^{+}$, while NAM inhibited this process (Figure 4D). The deacetylase-inactive SIRT1-H363Y was unable to deacetylate SIRT6.

As SIRT1 might interact with the SIRT6 N-terminus, we hypothesized that it might also deacetylate K33ac. While the acetylation level of SIRT6 was increased in $S_{\text {SIRT1 }}{ }^{--}$HEK293 cells, that of K33R was hardly affected (Figure 4E). Additionally, the acetylation level of SIRT6 K33R was minimally changed upon SIRT1 overexpression (Figure 4F), whereas that of K143/145R was downregulated upon ectopic SIRT1 (Figure S8A). These data support that K33ac is a target of SIRT1. By contrast, the SIRT1 acetylation level was relatively unaffected upon SIRT6 overexpression (Figure S8B). To further validate these findings, we synthesized a K33ac-containing peptide, and found that it effectively blocked the in vitro binding of SIRT6 to SIRT1 (Figure 4G). Of note, the GST pulldown assay suggested that the $\mathrm{N}$-terminus rather than the C-terminus of SIRT6 was responsible for its interaction with SIRT1. Together, these data suggest that SIRT1 deacetylates SIRT6 at K33. 

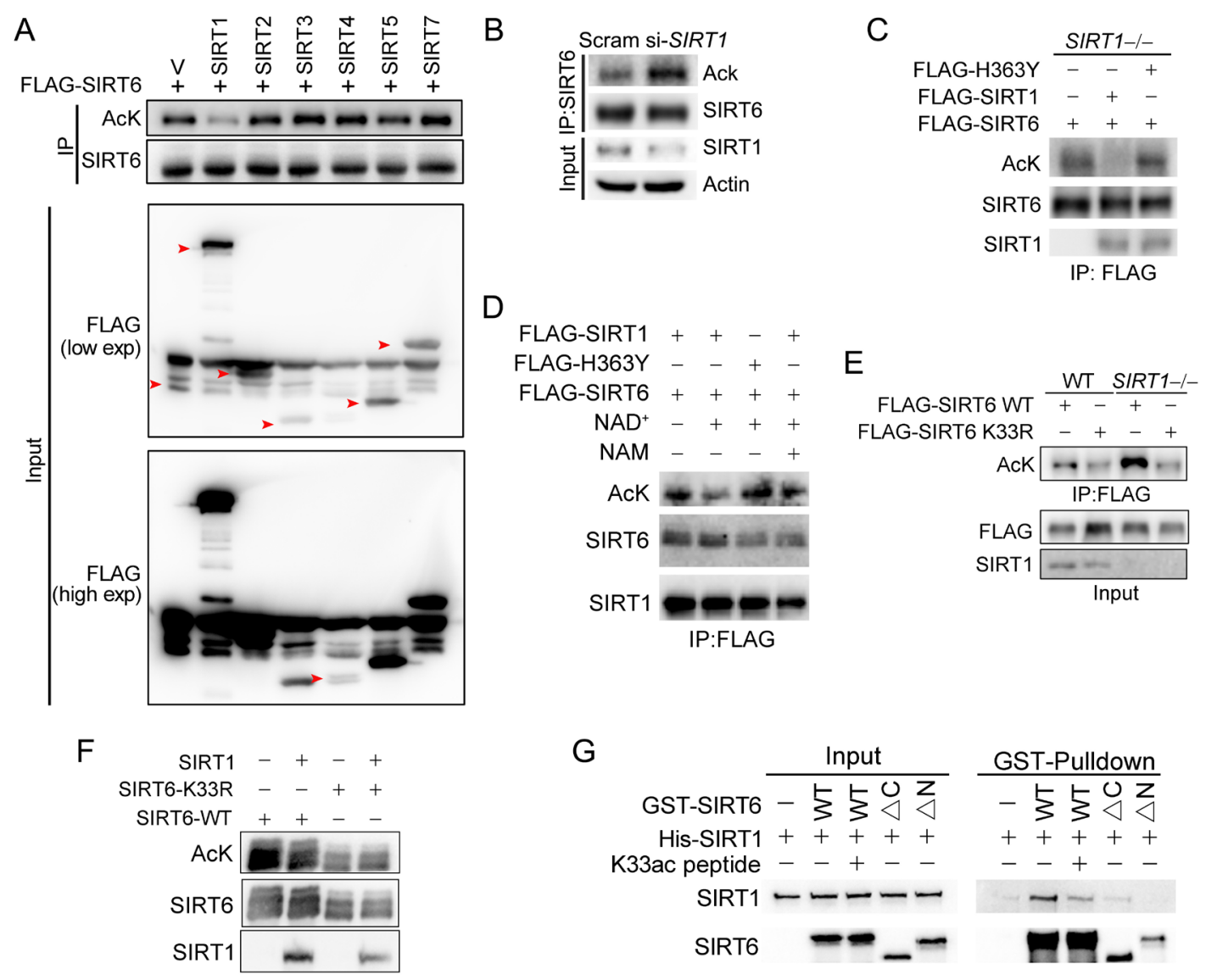

\section{Figure 4. SIRT1 deacetylates SIRT6 at K33}

(A) The acetylation level of FLAG-SIRT6 in HEK293 cells ectopically expressing SIRT1-5 and SIRT7.

(B) The acetylation level of endogenous SIRT6 in HEK293 cells treated si-SIRT1 or scramble (Scram) siRNAs.

(C) The acetylation level of FLAG-SIRT6 in SIRT1 $1^{-/}$cells reconstituted with SIRT1 or the enzyme-inactive SIRT1 H363Y.

(D) The acetylation level of FLAG-SIRT6 in the presence of SIRT1, H363Y, NAD ${ }^{+}$ $(500 \mu \mathrm{M})$ and/or NAM $(2 \mathrm{mM})$.

(E) The acetylation level of FLAG-SIRT6 and FLAG-SIRT6 K33R in $S I R T 1^{-/}$and WT HEK293 cells.

(F) The acetylation level of FLAG-SIRT6 and K33R in HEK293 cells with or without ectopic SIRT1.

(G) GST pulldown assay with GST-SIRT6 WT, $\Delta \mathrm{N}, \Delta \mathrm{C}$ and His-SIRT1 in the presence or absence of $10 \mu \mathrm{M}$ K33ac peptide [PEELERK(ac)VWELARL], which 
represents a 14-aa peptide containing acetylated SIRT6 K33.

\section{$\gamma \mathrm{H2AX}$ ensures SIRT6 retention surrounding DSBs}

$\gamma \mathrm{H} 2 \mathrm{AX}$ is dispensable for initial DSB recognition but serves as a platform for recruiting DDR factors (2). Because SIRT6 is enriched at DNA breaks, we next asked whether $\gamma \mathrm{H} 2 \mathrm{AX}$ is involved in SIRT6 recruitment. We thus performed a co-IP of endogenous SIRT6 in cells treated with or without camptothecin (CPT). Interestingly, $\mathrm{H} 2 \mathrm{AX}$ and $\gamma \mathrm{H} 2 \mathrm{AX}$ were detected in the anti-SIRT6 precipitates only when the cells were treated with CPT (Figure 5A,B). We also performed an in vitro pulldown assay with a biotin-labeled C-terminal $\gamma \mathrm{H} 2 \mathrm{AX}$ peptide or H2AX peptide. Consistently, GST-SIRT6 recognized the $\gamma \mathrm{H} 2 \mathrm{AX}$ peptide but not the H2AX peptide (Figure 5C). To identify the interacting domain, we purified various GST-SIRT6 truncation mutants. Peptide pulldown assay revealed that the N-terminus truncation was enough to abolish SIRT6 binding to $\gamma \mathrm{H} 2 \mathrm{AX}$ peptide, while the C-terminus truncation had a minimal effect (Figure 5D). We then investigated whether SIRT1-mediated deacetylation contributes to SIRT6 binding to $\gamma \mathrm{H} 2 \mathrm{AX}$. Here, the K33R mutant efficiently bound to $\gamma \mathrm{H} 2 \mathrm{AX}$ to a similar extent as WT SIRT6, but the binding was abolished in the case of K33Q (Figure 5E).

To investigate the functional relevance of this SIRT6- $\gamma \mathrm{H} 2 \mathrm{AX}$ interaction, we applied laser-induced DNA damage in MEFs lacking $H 2 A X$ and then tracked the distribution of SIRT6 by immunofluorescence microscopy. GFP-SIRT 6 was immediately recruited to DNA lesions in $H 2 A X^{+/+}$and $H 2 A X^{-/-}$MEFs (Figure 5F), implying that $\mathrm{H} 2 \mathrm{AX}$ is dispensable for initial SIRT6 recruitment of SIRT6. Interestingly, GFP-SIRT6 diminished from DNA lesions at $10 \mathrm{~min}$ after laser treatment in $H 2 A X^{-/}$MEFs but persisted in $\mathrm{H}_{2} \mathrm{AX}^{+/+}$cells. $\mathrm{H} 2 \mathrm{AX}$ is rapidly phosphorylated at serine 139 in response to DSBs (32). When we re-introduced H2AX WT, S139A and S139D into $H 2 A X^{-/-}$ MEFs, SIRT6 retention was restored in WT and S139D-re-expressing cells but not in S139A-re-expressing cells (Figure 5G). Together, these data indicate that SIRT6 recognizes $\gamma \mathrm{H} 2 \mathrm{AX}$ surrounding $\mathrm{DSBs}$ and that this recognition is enhanced by SIRT1-mediated deacetylation. 

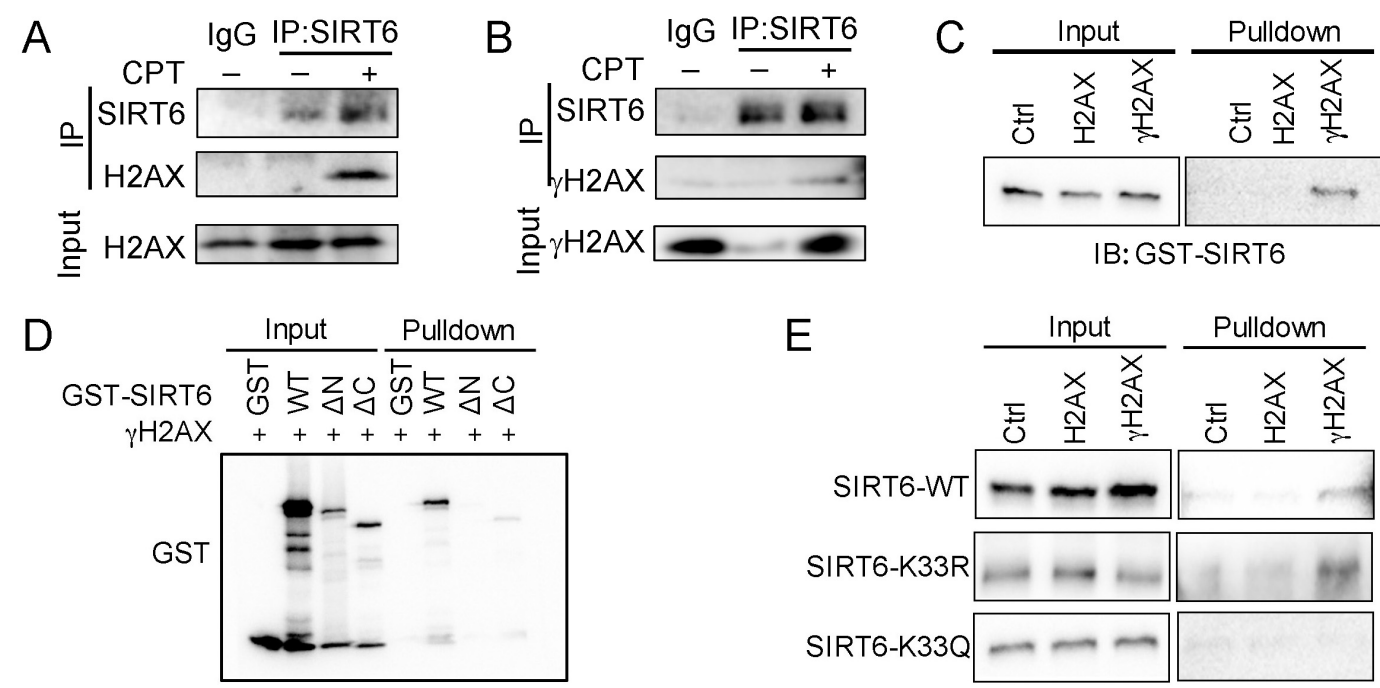

$\mathrm{F}$
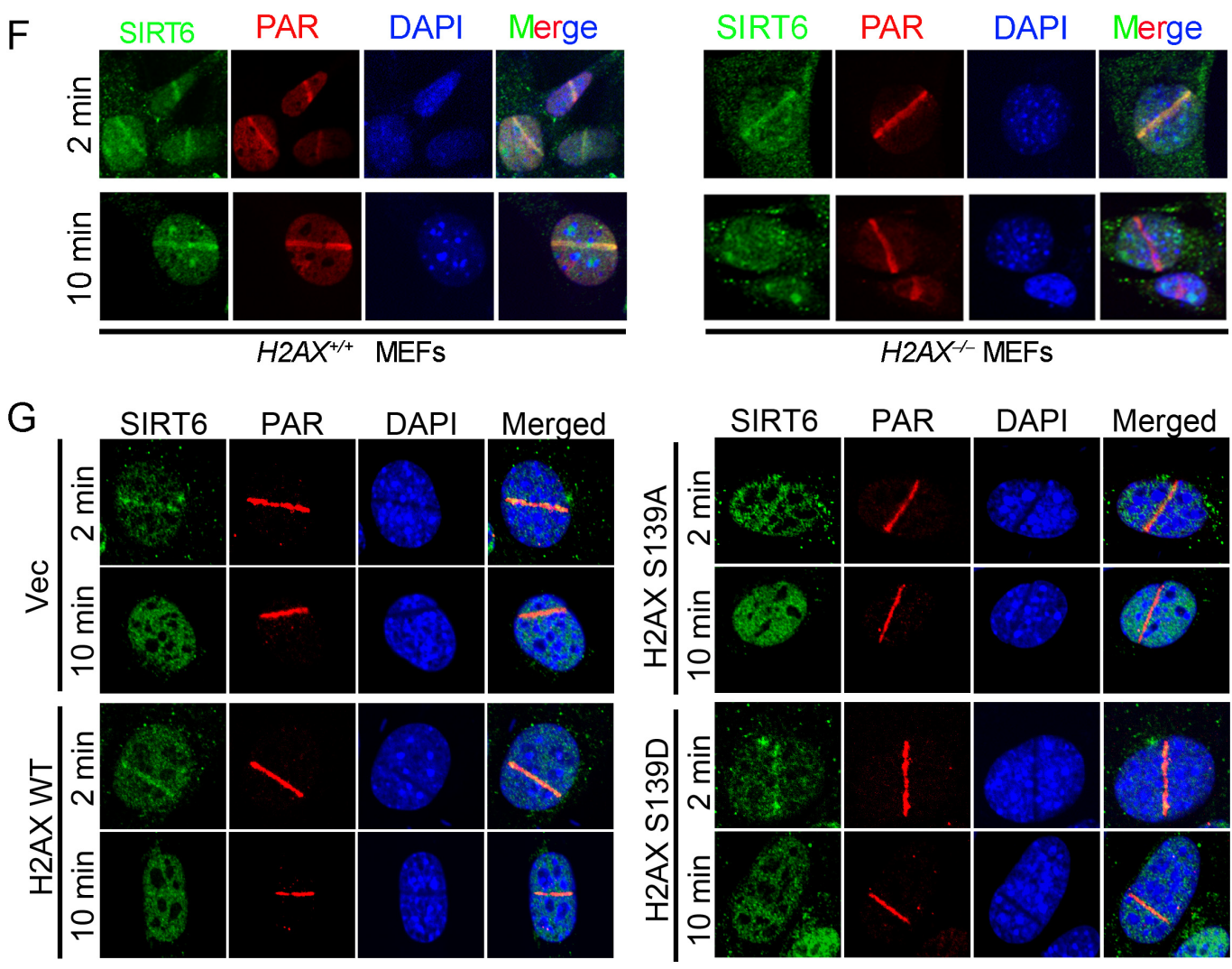

Figure 5. $\gamma \mathrm{H} 2 \mathrm{AX}$ is required for the chromatin retention of SIRT6.

(A,B) Representative western blots showing H2AX (A) and $\gamma$-H2AX (B) levels in anti-SIRT6 immunoprecipitates from HEK293 cells treated with or without camptothecin (CPT).

(C) Pulldown assay and western blot analysis of the interactions between GST-SIRT6 and $\gamma \mathrm{H} 2 \mathrm{AX}$ and $\mathrm{H} 2 \mathrm{AX}$ peptides.

(D) Pulldown assay and western blot analysis of the interactions between the $\gamma \mathrm{H} 2 \mathrm{AX}$ 
peptide, GST-SIRT6 WT and truncated GST-SIRT6 $\Delta \mathrm{N}$ and $\Delta \mathrm{C}$.

(E) Pulldown assay and western blot analysis of the interactions between the $\gamma \mathrm{H} 2 \mathrm{AX}$ peptide and GST-SIRT6 WT, K33R and K33Q.

$(\mathbf{F}, \mathbf{G})$ Laser MicroPoint analysis of SIRT6 recruitment in $H 2 A X^{+/+}$and $H 2 A X^{-/}$MEFs (F), and in $H 2 A X^{--}$MEFs reconstituted with H2AX WT, S139D mimicking hyper-phosphorylation or S139A mimicking hypo-phosphorylation (G). PAR immunostaining was used to identify the DNA damage site.

\section{SIRT1 and SIRT6 cooperatively promote DNA repair}

The physical interaction between SIRT1 and SIRT6 prompted us to investigate whether SIRT1 and SIRT6 cooperatively modulate the DDR and DNA repair. To do so, we combined the DR-GFP reporter system with a ChIP-PCR analysis. First, we found that FLAG-SIRT6 recruitment to the DSB vicinity was significantly reduced when SIRT1 was silenced by siRNA in HEK293 cells (Figures 6A,B). Then we monitored the dynamic recruitment of GFP-SIRT6 upon laser-induced DNA damage using a MicroPoint system. GFP-SIRT6 was rapidly recruited to DSBs in WT cells, but this process was largely deferred in Sirt ${ }^{-/-}$MEFs (Figures 6C,D), suggesting an indispensable role of SIRT1 in the initial recruitment of SIRT6 to DSBs. By contrast, SIRT1 recruitment to DSBs was relatively unaffected by SIRT6 downregulation, as determined by ChIP-PCR analysis (Figures 6E-G). 

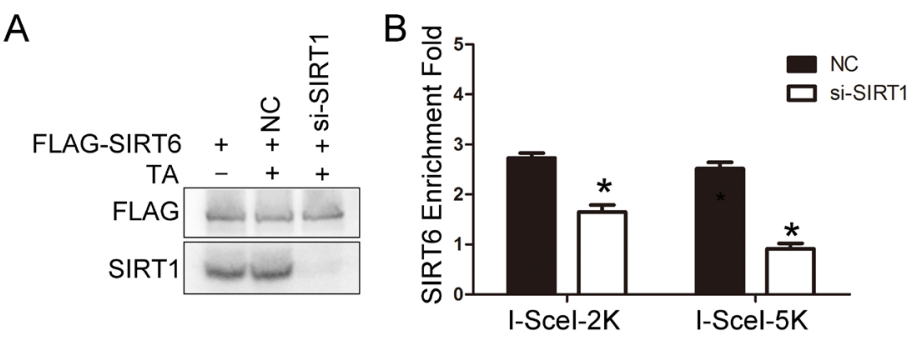

C
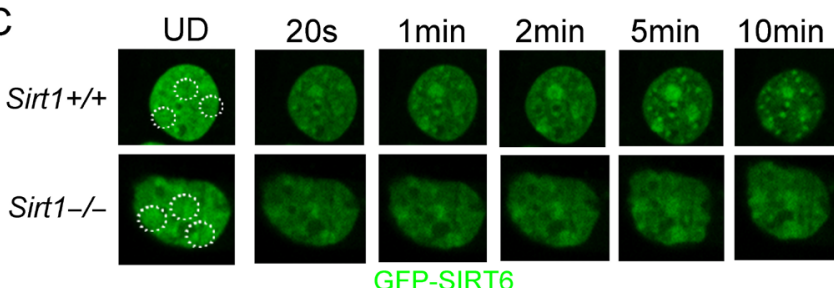

$\mathrm{F}$

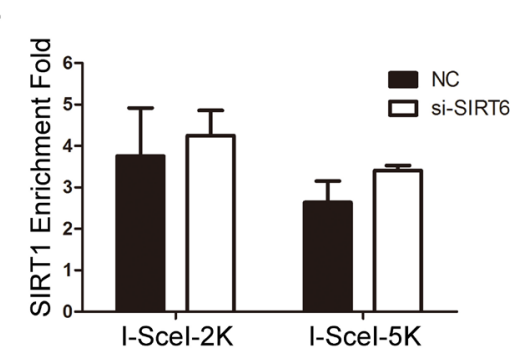

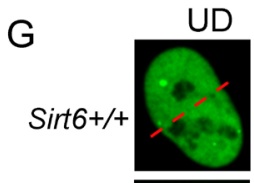

UD

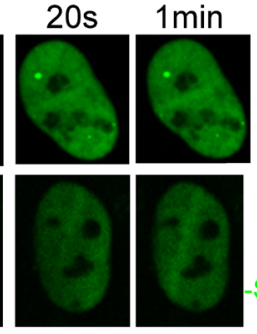

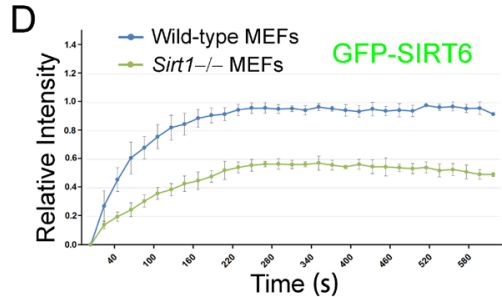

E
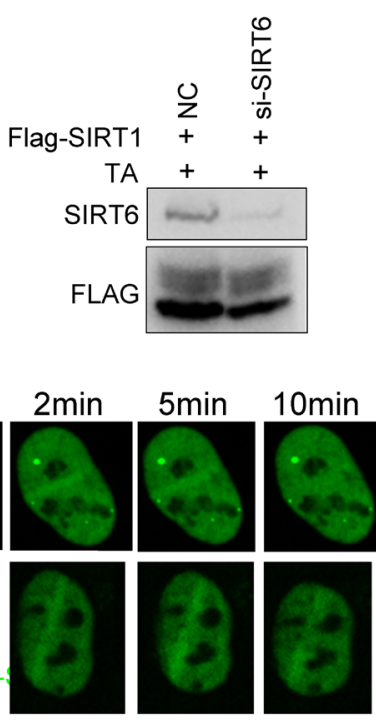

Figure 6. SIRT1 facilitates SIRT6 recruitment to chromatin during the DDR.

$(\mathbf{A}, \mathbf{B})$ ChIP-qPCR analysis of the SIRT6 levels in the vicinity of a DSB in cells treated with a SIRT1 siRNA (si-SIRT1) or a scrambled negative control (NC). The western blots show the FLAG-SIRT6 and SIRT1 protein levels.

(C,D) GFP-SIRT6 was introduced into Sirt1 ${ }^{+/+}$and Sirt ${ }^{-/-}$MEFs and the fluorescence signal was captured at various time points after laser-induced DNA damage. Representative images are shown $(\mathbf{C})$. The white dashed circles indicate the damage sites. The relative intensity was calculated in Image $J^{\circledR}(D)$.

(E,F) ChIP-qPCR analysis of the SIRT1 levels in the vicinity of a DSB in cells treated with SIRT6 siRNA (si-SIRT6) or NC. The western blots show the FLAG-SIRT1 and SIRT6 protein levels.

(G) GFP-SIRT1 was introduced into Sirt6 ${ }^{+/+}$and Sirt6 ${ }^{-/}$MEFs and the fluorescence signal was captured after laser-induced damage at various time points. Representative images are shown. The red dashed lines indicate laser-induced damage sites. 
In our final set of assays, we wanted to determine the function of SIRT6 deacetylation in DNA repair. We found that SIRT6 acetylation was significantly decreased upon CPT treatment, but that the effects of CPT were abolished in the presence of SIRT6 K33R or in the absence of SIRT1 (Figure 7A). These data imply that SIRT6 is deacetylated by SIRT1 upon DNA damage. We then examined the effects of the SIRT6 mutants on DNA repair by comet assay, which assesses the DNA repair ability at the single cell level. We overexpressed SIRT6 K33R or K33Q in SIRT6 ${ }^{-/-}$cells and then examined the DNA repair efficacy. Here, overexpression of SIRT6 significantly enhanced DNA repair efficacy upon CPT treatment, while K33Q or H133Y lost the ability. By contrast, SIRT6 K33R overexpression promoted DNA repair to an extent comparable to WT (Figure 7B). An HR assay showed that SIRT6 WT and K33R but neither K33Q nor H133Y enhanced HR capacity (Figure 7C). Furthermore, compared to SIRT6 WT and K33R, K33Q significantly inhibited the colony forming capacity of transfected Hela cells (Figure 7D). Upon ionized radiation, while both SIRT6 WT and K33R enhanced cell survival, K33Q and H133Y inhibited it (Figure 7E). These data implicate that SIRT6 deacetylation at K33 is indispensable for DNA repair.

SIRT1 regulates DNA repair (6). To elucidate the synergistic effects of SIRTs in DNA repair, we examined whether SIRT6 hyper-acetylation is responsible for the defective

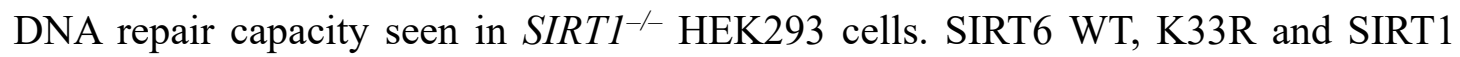
overexpression rescued the defective DNA repair imposed by the SIRT1 deficiency, while SIRT6 K33Q and H133Y had minimal rescue effect (Figure 7F). Altogether, these data implicate a synergistic action between SIRT1 and SIRT6 in regulating the DDR and DNA repair. We thus propose a model by which SIRT6 is deacetylated by SIRT1 at K33, thus promoting its polymerization and recognition of DSBs; SIRT6 that is deacetylated at $\mathrm{K} 33$ anchors to $\gamma \mathrm{H} 2 \mathrm{AX}$, allowing retention on the chromatin flanking the DSBs and subsequent remodeling via deacetylating H3K9ac and H3K56ac (Figure 7G). 
A

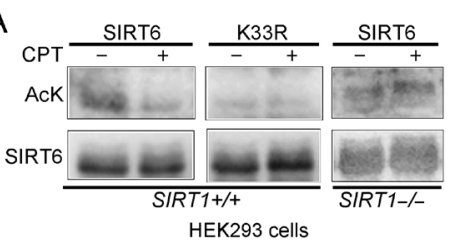

C

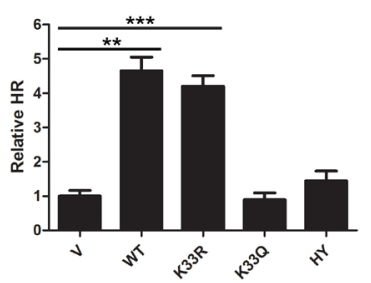

$\mathrm{F}$

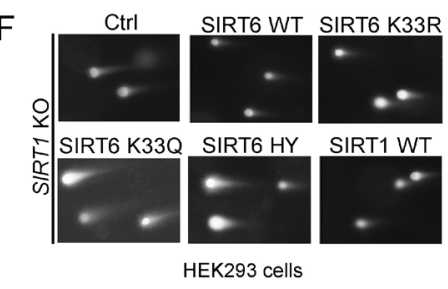

B
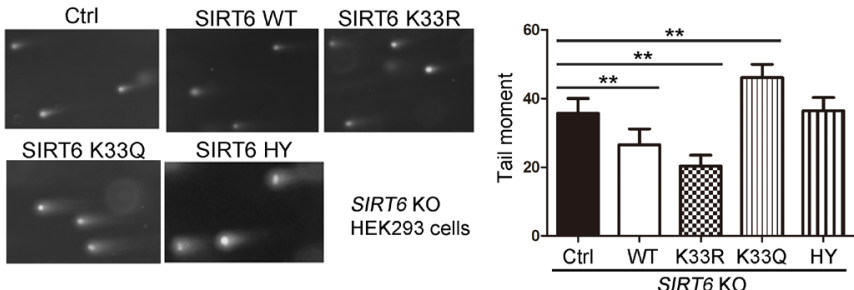

$\mathrm{E}$

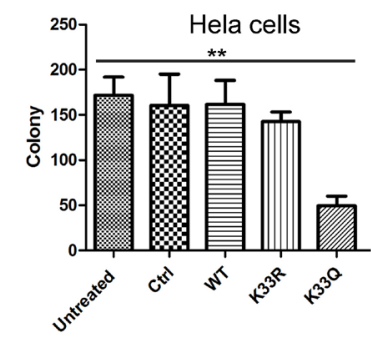

G
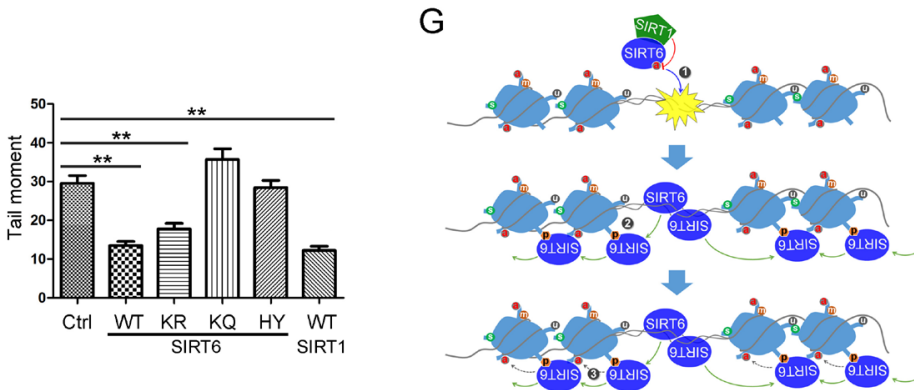

Figure 7. SIRT6 rescues DNA repair defects caused by a SIRT1 deficiency.

(A) The acetylation level of SIRT6 WT and K33R in SIRT1 ${ }^{+/+}$and SIRT1 ${ }^{-/-}$HEK293 cells treated or not with CPT $(1 \mu \mathrm{M})$ for $1 \mathrm{~h}$.

(B) Comet assay in FLAG-SIRT6, K33R, K33Q and HY reconstituted SIRT6 KO cells treated with CPT for $1 \mathrm{~h}$. The data represent the means \pm s.e.m. $* * p<0.01$.

(C) HR assay in U2OS cells ectopically expressing FLAG-SIRT6, K33R, K33Q or HY. The relative HR value was normalized to the vector control. The data represent the means \pm s.e.m. $* * * p<0.001$. $* * p<0.01$.

(D) Colony-forming assay in Hela cells ectopically expressing FLAG-SIRT6, K33R or K33Q. The data represent the means \pm s.e.m. ${ }^{* *} p<0.01$.

(E) Colony-forming assay in Hella cells stable expressing SIRT6 WT, K33R, K33Q or HY after radiation at indicated dose. The data represent the means \pm s.e.m. ${ }^{*} p<$ 0.05 .

(F) Comet assay in SIRT1 ${ }^{-/}$HEK293 cells transfected with FLAG-SIRT6, K33R, K33Q, HY and SIRT1 and treated with CPT for $1 \mathrm{~h}$. The data represent the means \pm s.e.m. $* * p<0.01$. 
(G) A working model: (a) SIRT6 is deacetylated by SIRT1 at K33, which promotes SIRT6 polymerization and recognition of DSBs. (b) Beyond DSBs, K33-deacetylated SIRT6 anchors to $\gamma \mathrm{H} 2 \mathrm{AX}$ and expands on local chromatin flanking DSBs. (c) SIRT6 mediates local chromatin remodeling via deacetylating H3K9ac.

\section{Discussion}

The DDR is a highly orchestrated process that is initiated by DNA break-sensing (1). While the MRN complex (33), Ku complex (28), RPA (34) and PARP1 $(35,36)$ are all known to directly recognize DSBs, sirtuins are among the earliest factors to be recruited to DSBs $(17,18)$ and facilitate PARP1 recruitment (7). Consistent with published data (23), we found that SIRT6 oligomerizes and recognizes DSBs via a DSB-binding pocket generated by the N-termini and C-termini of two adjacent molecules. This finding is consistent with another report showing that both the $\mathrm{N}$-termini and C-termini are essential for the chromatin association of SIRT6 (26). Using a super-resolution fluorescent particle tracking method, Yang et al. recently found that PARP1 binding to DSBs happens earlier than SIRT6 binding (37). One possible explanation is that PARP1 is first recruited to DSBs; then, SIRTs are later recruited directly by DSBs and facilitate PARP1 stabilization and expansion in the surrounding region.

The sirtuin family members share similar functions in the DDR and in DNA repair; upon DNA damage, both SIRT1 and SIRT6 are rapidly mobilized to DSBs $(7,17,18)$. SIRT1 redistributes on chromatin and deacetylates XPA, NBS1 and Ku70 to promote DNA repair (8-11). SIRT6 mono-ribosylates PARP1 to enhance its activity (16), and facilitates the subsequent recruitment of SNF2H, H2AX and DNA-PKcs (12-15). Here we revealed a synergistic action between two nuclear SIRTs, whereby SIRT1 deacetylates SIRT6 to promote its mobilization to DSBs. A SIRT6 K33R mutant, mimicking hypo-acetylated SIRT6, can rescue DNA repair defects in SIRT1 null cells. Interestingly, SIRT6 phosphorylation at S10 by JNK promotes subsequent recruitment itself and PARP1 upon oxidative stress, also supporting an essential role of the SIRT6 $\mathrm{N}$ terminus for DSB-recruitment (15). Consistent with the cooperative action between SIRT1 and SIRT6, independent studies have revealed an interaction between SIRT1 and SIRT7, showing that SIRT1 recruits SIRT7 to promote cancer cell metastasis (38), 
and that SIRT1 and SIRT7 antagonistically regulate adipogenesis (39).

The acetylation levels of H3K9 and H3K56 decrease upon detecting DNA damage and then return to basal levels (31). SIRT1 and SIRT6 are H3K9ac and H3K56ac deacetylases; both are recruited to DSBs upon DNA damage, indicating that SIRT1 and/or SIRT6 might contribute to reducing H3K9ac and H3K56ac levels. Although mechanistically unclear, H3K9ac and $\mathrm{H} 3 \mathrm{~K} 56 \mathrm{ac}$ levels negatively correlate with $\gamma \mathrm{H} 2 \mathrm{AX}$ levels (40). In this study, we found that while $\gamma \mathrm{H} 2 \mathrm{AX}$ is not required for initial SIRT6 recruitment, it is indispensable for retaining SIRT6 on the local chromatin surrounding DSBs. This finding is consistent with reports that $\gamma \mathrm{H} 2 \mathrm{AX}$ is dispensable for initial reorganization of DNA breaks but rather serves as a platform to stabilize repair factors, such as NBS1, 53BP1 and BRCA1 (2). SIRT6 deacetylates $\mathrm{H} 3 \mathrm{~K} 9 \mathrm{ac}$ and $\mathrm{H} 3 \mathrm{~K} 56 \mathrm{ac}$ surrounding DSBs, in this way bridging $\gamma \mathrm{H} 2 \mathrm{AX}$ to chromatin remodeling. Together, these findings provide a scenario as to how $\gamma \mathrm{H} 2 \mathrm{AX}$ and histone modifiers coordinate to amplify the DDR.

SIRT6 and SNF2H cooperate to stabilize $\gamma \mathrm{H} 2 \mathrm{AX}$ foci (41). Here we found that $\gamma \mathrm{H} 2 \mathrm{AX}$ in-fact anchors SIRT6 to DSBs, providing a positive feedback regulatory loop between SIRT6 and $\gamma \mathrm{H} 2 \mathrm{AX}$. This finding is consistent with reports showing a distinct reduction of $\gamma \mathrm{H} 2 \mathrm{AX}$ and an improper DDR in Sirt $6^{-/-}$and Sirt $1^{-/-}$cells. Recent work also suggests that an electrostatic force between a negatively charged phosphate group and a positively charged lysine groups is a novel form of protein-protein interaction (27). We thus consider it plausible to speculate that (de)acetylation might act as a switch to modulate such an interaction between SIRT6 and $\gamma \mathrm{H} 2 \mathrm{AX}$.

Known as longevity-associated genes, SIRT6 and SIRT1 are redundant in DNA repair but not replaceable. In this study, we have identified that SIRT6 directly binds to DNA breaks and have elucidated a physical and functional interaction between SIRT6 and SIRT1. SIRT6 rescues DNA repair defects imposed by SIRT1 deficiency. Overall, these data highlight a synergistic action of nuclear SIRTs in the spatiotemporal regulation of the DDR and DNA repair.

\section{Materials and Methods}

\section{Antibodies, Oligos and Plasmids}


The commercial antibodies used in this study included: SIRT6, SNF2H, pan-AcK, H3 and $\gamma \mathrm{H} 2 \mathrm{AX}$ (Abcam), H2AX, SIRT1 and GST (CST), H3K9ac and H3K56ac (Millipore), SIRT6 (Novus), Tubulin and FLAG (Sigma).

The following oligos (Genewiz) were used for RNA interference:

siSIRT6, 5'-AAGAAUGUGCCAAGUGUAAGA-3';

siSIRT1, 5'-ACUUUGCUGUAACCCUGUA-3'.

The following primers were used for ChIP qPCR:

I-SceI- 2k-F, 5'-GCCCATATATGGAGTTCCGC-3';

I-SceI-2k-R, 5'-GGGCCATTTACCGTCATTG-3';

I-SceI-5k-F, 5'-GTTGCCGGGAAGCTAGAGTAAGTA-3';

I-SceI-5k-R, 5'-TTGGGAACCGGAGCTGAATGAA-3'.

The following gRNA sequences were used for CRISPR/Cas9 gene editing:

Hu Sirt6: gRNA-F, 5'-CACCGGCTGTCGCCGTACGCGGACA-3';

gRNA-R, 5'-AAACTGTCCGCGTACGGCGACAGCC-3'.

Hu Sirt1: gRNA-F, 5'-CACCGATAGCAAGCGGTTCATCAGC-3'

Human SIRT6 was cloned into pCDNA3.1 with a FLAG tag (Invitrogen, USA); a $3 \times$ FLAG-SIRT1 and DR-GFP plasmids were obtained from Addgene. SIRT $6 \Delta \mathrm{C}$ and $\Delta \mathrm{N}$ were amplified with specific primers and cloned into pKH3HA (Addgene) and pGex vectors (GE Healthcare Life Sciences). The SIRT6 KR, KQ and HY mutants were obtained by converting SIRT6 lysine 33 to arginine (KR), or to glutamine (KQ) and SIRT6 133 histidine to tyrosine (HY) via site-directed mutagenesis, as described below.

\section{Site-directed mutagenesis}

The primers used for mutagenesis were designed using the online Quick Change Primer Design Program provided by Agilent Technologies. The mutagenesis was performed using Pfu DNA polymerase (Agilent) and $300 \mathrm{ng}$ plasmid template, according to the manufacturer's instructions. The PCR product was digested with $D p n I$ endonuclease for $1 \mathrm{~h}$ at $37^{\circ} \mathrm{C}$, before transformation and sequencing.

The following primers were used to generate the SIRT6 KR, KQ and HY mutants: 
KR forward: 5'-GGAGCTGGAGCGGAGGGTGTGGGAACT-3'

KR reverse: 5'-AGTTCCCACACCCTCCGCTCCAGCTCC-3'

KQ forward: 5'-GGAGCTGGAGCGGCAGGTGTGGGAACT-3'

KQ reverse: 5'-AGTTCCCACACCTGCCGCTCCAGCTCC-3'

HY forward: 5'-ACAAACTGGCAGAGCTCTACGGGAACATGTTTGTG-3'

HY reverse: 5'-CACAAACATGTTCCCGTAGAGCTCTGCCAGTTTGT-3'

\section{Immunoprecipitation}

HEK293T cells were transfected with the indicated plasmids using Lipofetamine ${ }^{\circledR} 3000$ (Invitrogen, USA), according to the manufacturer's instructions. The cells were lysed $48 \mathrm{~h}$ post-transfection in lysis buffer [50 mM Tris-HCl, $\mathrm{pH}$ 7.4, $200 \mathrm{mM} \mathrm{NaCl}, 0.2 \%$ NP40, 10\% glycerol, $1 \mathrm{mM} \mathrm{NaF}, 1 \mathrm{mM}$ Sodium butyrate, 10 $\mathrm{mM}$ Nicotinamide and a Complete protease inhibitor cocktail (Roche)]. The cell extracts were incubated with anti-FLAG M2 monoclonal antibody-conjugated agarose beads (Sigma) at $4^{\circ} \mathrm{C}$ overnight with rotation. The immunoprecipitates were boiled IN $2 \times$ laemmli buffer and then analyzed by western blotting.

\section{Chromatin Immunoprecipitation (ChIP)}

I-SceI-GR assays were performed as previously described (30). Hela cells stably transfected with DR-GFP were transiently transfected with RFP-I-SceI-GR together with FLAG-SIRT6, KR, KQ or HY. The cells were treated with $10^{-7} \mathrm{M}$ triamcinolone acetonide (TA, Sangon, Shanghai) for $20 \mathrm{~min}, 48 \mathrm{~h}$ after transfection, and then fixed with $1 \%$ paraformaldehyde at $37^{\circ} \mathrm{C}$ for $10 \mathrm{~min}$ to crosslink the chromatin. The reaction was stopped upon the addition of $0.125 \mathrm{M}$ glycine. The chromatin was sonicated to 200 bps $\sim 600$ bps and incubated with the indicated antibodies. After de-cross linking, the ChIP-associated DNA was isolated and analyzed by quantitative real-time PCR (qRT-PCR).

\section{Comet assay}

A comet assay was performed as previously described (42). Briefly, after CPT treatment, the cells were digested into a single cell suspension, mixed with $1 \%$ agarose at the density of $1 \times 10^{5}$, coated on the slide and then incubated in lysis buffer ( $2 \%$ sarkosyl, $0.5 \mathrm{M} \mathrm{Na} 2$ EDTA, $0.5 \mathrm{mg} / \mathrm{ml}$ proteinase $\mathrm{K}$ ) overnight at $37^{\circ} \mathrm{C}$. The slides were incubated with $\mathrm{N} 2$ buffer ( $90 \mathrm{mM}$ Tris, $90 \mathrm{mM}$ boric acid and $2 \mathrm{mM} \mathrm{Na} 2 \mathrm{EDTA}$ ) 
and subjected to electrophoresis for $25 \mathrm{~min}$ at $0.6 \mathrm{~V} / \mathrm{cm}$. The slides were then incubated in staining solution containing $2.5 \mu \mathrm{g} / \mathrm{ml}$ propidium iodide for $30 \mathrm{~min}$ at room temperature. Images were captured under a fluorescent microscope.

\section{Cell fractionation}

Cells were scraped and washed with cold PBS. The cell pellet was resuspended in nuclei lysis buffer (10 mM HEPES, $10 \mathrm{mM} \mathrm{KCl}, 1.5 \mathrm{mM} \mathrm{MgCl}, 0.34 \mathrm{M}$ sucrose, $10 \%$ glycerol, $1 \mathrm{mM}$ DTT, $0.1 \%$ TrionX-100.) for $10 \mathrm{~min}$ on ice and then centrifuged at $1300 \mathrm{~g}$ for $10 \mathrm{~min}$. The pellet was resuspended in lysis buffer ( $3 \mathrm{mM}$ EDTA, 0.2 mM EGTA, $1 \mathrm{mM}$ DTT) for $10 \mathrm{~min}$ on ice and centrifuged at $1700 \mathrm{~g}$ for $10 \mathrm{~min}$. The pellet was saved as the chromatin fraction.

\section{MicroPoint ${ }^{\circledR}$ laser irradiation and microscopy}

U2OS cells or MEFs were seeded on a dish with a thin glass bottom (NEST), then locally irradiated with a $365 \mathrm{~nm}$ pulsed UV laser (16 Hz pulse, 56\% laser output), generated by the MicroPoint Laser Illumination and Ablation System (Andor $\AA$; power supply TPES24-T120MM, Laser NL100, 24V 50W), which is coupled to the fluorescence path of the Nikon A1 confocal imaging system (TuCam). Fluorescent protein recruitment and retention were continuously monitored by time-lapse imaging every $20 \mathrm{~s}$ for $10 \mathrm{~min}$. The fluorescence intensity was quantified at each time-point using Fiji (image J) software.

\section{CRISPR/Cas9-mediated gene editing}

CRISPR/Cas9-mediated gene editing was conducted as described (Ran et al., 2013). Briefly, a pX459 vector (Addgene\#48139) was digested with BbsI and ligated with annealed oligonucleotides. The constructs containing the target gRNAs were transfected into HEK293T cells with Lipofetamine3000® (Invitrogen). The cells were selected for 5 days with puromycin $24 \mathrm{~h}$ after transfection. Single clones were picked for sequencing.

\section{Peptide pulldown assay}

The $\mathrm{C}$ termini of H2AX (BGKKATQASQEY) and $\gamma \mathrm{H} 2 \mathrm{AX}$ (BGKKATQApSQEY) were synthesized and conjugated with biotin (GL Biochem, Shanghai). For one reaction, $1 \mu \mathrm{g}$ biotinylated peptides was incubated with $1 \mu \mathrm{g}$ GST-SIRT6 in binding buffer (50 mM Tirs-HCl, $200 \mathrm{mM} \mathrm{NaCl}, 0.05 \%$ NP40) overnight at $4^{\circ} \mathrm{C}$. Streptavidin 
Sepharose beads (GE) was then used to pulldown the peptide and protein complexes for $1 \mathrm{~h}$ at $4^{\circ} \mathrm{C}$, and the samples were analyzed by western blotting.

\section{Immunofluorescence staining}

The cells were washed with PBS and fixed with 4\% formaldehyde for $20 \mathrm{~min}$, followed by permeabilization with cold methanol $\left(-20^{\circ} \mathrm{C}\right)$ for $5 \mathrm{~min}$ and blocking with $5 \%$ BSA for $30 \mathrm{~min}$. Then, the cells were incubated with primary antibodies (SIRT1, 1:200 dilution in 1\% BSA; $\gamma \mathrm{H} 2 \mathrm{AX}, 1: 500$ dilution in 1\% BSA; SIRT6, 1:200 dilution in $1 \% \mathrm{BSA}$ ) for $1 \mathrm{~h}$ and secondary antibodies (donkey anti-rabbit IgG Alexa Fluor 594 and donkey anti-mouse IgG FITC from Invitrogen, 1:500 dilution in1\% BSA) for $1 \mathrm{~h}$ at room temperature in the dark. The cells were then co-stained with DAPI (Invitrogen) and observed under a fluorescent microscope.

\section{HR assay}

U2OS cells stably transfected with DR-GFP were transfected with HA-I-SceI together with Flag-SIRT6 WT, K33R, K33Q or HY. After transfection for 48 h, the cells were harvested and the GFP positive cell ratio per $10^{4}$ cells was analyzed by flow cytometry (BD Biosciences). The relative HR efficiency was normalized to the vector control.

\section{Colony formation assay}

Hella cells were seeded into six-well plates $24 \mathrm{~h}$ after transfection in defined numbers. Then, $24 \mathrm{~h}$ after re-plating, the cells were exposed to increasing amounts of ionizing radiation delivered by an X-Rad 320 irradiator (Precision X-Ray Inc. N. Branford, CT, USA). Fresh media was added after 7 days. Colonies containing at least 50 cells (10-14 days) were fixed with $20 \%$ methanol and stained with crystal violet and analyzed.

\section{DNA pulldown assay}

The DNA binding assay was performed as previously described (43). Briefly, a biotin-conjugated DNA duplex 220 bp in size was generated by PCR amplification using biotin-labeled primers and a I-SceI plasmid as a template.

For the DNA pulldown assay, 10 pmol biotinylated DNA duplex was incubated with $0.5 \mu \mathrm{g}$ of the indicated recombinant proteins in $300 \mu$ binding buffer $(10 \mathrm{mM}$ Tris-Cl pH7.5, $100 \mathrm{mM} \mathrm{NaCl}, 0.01 \% \mathrm{NP} 40$ and $10 \%$ glycerol) overnight at $4^{\circ} \mathrm{C}$. Streptavidin 
Sepharose beads (GE) were added the next day, and incubated for another $1 \mathrm{~h}$ with the samples. The beads were then collected and washed with binding buffer three times. The beads were subsequently boiled in $2 \times$ laemmli buffer and analyzed by western blotting.

For linear and circular DNA competition assays, the ratios of the non-biotin labeled linear/circular DNA to the biotin DNA duplex were 5:1 or 10:1. Linear DNA was generated by PCR amplification using non-biotin-labeled primers, and circular DNA was obtained by cloning a PCR product into the pCDNA 3.1 plasmid (Invitrogen, USA).

The following sequences were used for PCR:

Forward, 5'-TACGGCAAGCTGACCCTGAA-3'

Reverse, 5'-CGTCCTCCTTGAAGTCGATG-bio-3'

\section{FP assay}

SIRT1, SIRT6 and SIRT7 recombinant proteins were purified in vitro, and incubated with a FAM-conjugated DNA duplex $(20 \mathrm{nM})$ for $30 \mathrm{~min}$ on ice at the indicated concentration. The FP value of each sample was measured on 96 plates using a Multimode Plate Reader VictorTM X5 (PerkinElmer, USA) with an excitation wavelength of $480 \mathrm{~nm}$ and an emission wavelength of $535 \mathrm{~nm}$. Curve fitting was performed in GraphPad ${ }^{\circledR}$ prism.

\section{Statistical analysis}

Statistical analyses were conducted using two-tailed Student's t-test between two groups. All data are presented as mean \pm S.D. or mean \pm S.E.M. as indicated, and a $p$ value $<0.05$ was considered statistically significant. Independent experiments were performed in triplicates.

\section{Acknowledgements}

We thank Dr Linyu Lu (Zhejiang University, China) for providing $H 2 A X^{-/}$MEFs. This project was supported by research grants from the National Key R\&D Program of China (2017YFA0503900), the National Natural Science Foundation of China (91849208, 81972602, 81702909, 81871114, 81601215), the National Natural Science Foundation of Guangdong Province (2015A030308007, 2017B030301016), Shenzhen Science and Technology Innovation Commission 
(ZDSYS20190902093401689,

KQJSCX20180328093403969,

JCYJ20180507182044945), the Youth Foundation of Tianjin Medical University

Cancer Institute and Hospital (NO. B1714), and Tianjin Municipal Science

Foundation for Youths (NO. 18JCQNJC79800). The authors would like to thank Dr.

Jessica Tamanini (ETediting, Shenzhen University) for editing the manuscript prior to submission

\section{Competing interests}

The authors declare no competing interests.

\section{REFERENCES}

1. Ciccia, A. and Elledge, S.J. (2010) The DNA damage response: making it safe to play with knives. Mol Cell, 40, 179-204.

2. Celeste, A., Fernandez-Capetillo, O., Kruhlak, M.J., Pilch, D.R., Staudt, D.W., Lee, A., Bonner, R.F., Bonner, W.M. and Nussenzweig, A. (2003) Histone H2AX phosphorylation is dispensable for the initial recognition of DNA breaks. Nat. Cell Biol., 5, 675-679.

3. Price, B.D. and D'Andrea, A.D. (2013) Chromatin remodeling at DNA double-strand breaks. Cell, 152, 1344-1354.

4. Houtkooper, R.H., Pirinen, E. and Auwerx, J. (2012) Sirtuins as regulators of metabolism and healthspan. Nature reviews Molecular cell biology, 13, 225-238.

5. Mostoslavsky, R., Chua, K.F., Lombard, D.B., Pang, W.W., Fischer, M.R., Gellon, L., Liu, P., Mostoslavsky, G., Franco, S. and Murphy, M.M. (2006) Genomic instability and aging-like phenotype in the absence of mammalian SIRT6. Cell, 124, 315-329.

6. Wang, R.-H., Sengupta, K., Li, C., Kim, H.-S., Cao, L., Xiao, C., Kim, S., Xu, X., Zheng, Y. and Chilton, B. (2008) Impaired DNA damage response, genome instability, and tumorigenesis in SIRT1 mutant mice. Cancer cell, 14, 312-323.

7. Vazquez, B.N., Thackray, J.K., Simonet, N.G., Kane - Goldsmith, N., Martinez Redondo, P., Nguyen, T., Bunting, S., Vaquero, A., Tischfield, J.A. and Serrano, L. (2016) SIRT7 promotes genome integrity and modulates non - homologous end joining DNA repair. The EMBO journal, e201593499.

8. Fang, E.F., Kassahun, H., Croteau, D.L., Scheibye-Knudsen, M., Marosi, K., Lu, H., Shamanna, R.A., Kalyanasundaram, S., Bollineni, R.C., Wilson, M.A. et al. (2016) NAD+ Replenishment Improves Lifespan and Healthspan in Ataxia Telangiectasia Models via Mitophagy and DNA Repair. Cell metabolism, 24, 566-581.

9. Yuan, Z., Zhang, X., Sengupta, N., Lane, W.S. and Seto, E. (2007) SIRT1 regulates the function of the Nijmegen breakage syndrome protein. Mol. Cell, 27, 149-162.

10. Fan, W. and Luo, J. (2010) SIRT1 regulates UV-induced DNA repair through deacetylating XPA. Molecular cell, 39, 247-258.

11. Jeong, J., Juhn, K., Lee, H., Kim, S.H., Min, B.H., Lee, K.M., Cho, M.H., Park, G.H. 
and Lee, K.H. (2007) SIRT1 promotes DNA repair activity and deacetylation of Ku70. Exp Mol Med, 39, 8-13.

12. Van Meter, M., Simon, M., Tombline, G., May, A., Morello, Timothy D., Hubbard, Basil P., Bredbenner, K., Park, R., Sinclair, David A., Bohr, Vilhelm A. et al. (2016) JNK Phosphorylates SIRT6 to Stimulate DNA Double-Strand Break Repair in Response to Oxidative Stress by Recruiting PARP1 to DNA Breaks. Cell Reports, 16, 2641-2650.

13. Atsumi, Y., Minakawa, Y., Ono, M., Dobashi, S., Shinohe, K., Shinohara, A., Takeda, S., Takagi, M., Takamatsu, N., Nakagama, H. et al. (2015) ATM and SIRT6/SNF2H Mediate Transient H2AX Stabilization When DSBs Form by Blocking HUWE1 to Allow Efficient $\gamma \mathrm{H} 2 \mathrm{AX}$ Foci Formation. Cell Reports, 13, 2728-2740.

14. McCord, R.A., Michishita, E., Hong, T., Berber, E., Boxer, L.D., Kusumoto, R., Guan, S., Shi, X., Gozani, O., Burlingame, A.L. et al. (2009) SIRT6 stabilizes DNA-dependent protein kinase at chromatin for DNA double-strand break repair. Aging (Albany NY), 1, 109-121.

15. Van Meter, M., Simon, M., Tombline, G., May, A., Morello, T.D., Hubbard, B.P., Bredbenner, K., Park, R., Sinclair, D.A. and Bohr, V.A. (2016) JNK Phosphorylates SIRT6 to Stimulate DNA Double-Strand Break Repair in Response to Oxidative Stress by Recruiting PARP1 to DNA Breaks. Cell Reports, 16, 2641-2650.

16. Mao, Z., Hine, C., Tian, X., Van Meter, M., Au, M., Vaidya, A., Seluanov, A. and Gorbunova, V. (2011) SIRT6 promotes DNA repair under stress by activating PARP1. Science, 332, 1443-1446.

17. Dobbin, M.M., Madabhushi, R., Pan, L., Chen, Y., Kim, D., Gao, J., Ahanonu, B., Pao, P.-C., Qiu, Y. and Zhao, Y. (2013) SIRT1 collaborates with ATM and HDAC1 to maintain genomic stability in neurons. Nat. Neurosci., 16, 1008-1015.

18. Toiber, D., Erdel, F., Bouazoune, K., Silberman, D.M., Zhong, L., Mulligan, P., Sebastian, C., Cosentino, C., Martinez-Pastor, B. and Giacosa, S. (2013) SIRT6 recruits $\mathrm{SNF} 2 \mathrm{H}$ to DNA break sites, preventing genomic instability through chromatin remodeling. Mol. Cell, 51, 454-468.

19. Gil, R., Barth, S., Kanfi, Y. and Cohen, H.Y. (2013) SIRT6 exhibits nucleosome-dependent deacetylase activity. Nucleic Acids Res, 41, 8537-8545.

20. Tong, Z., Wang, M., Wang, Y., Kim, D.D., Grenier, J.K., Cao, J., Sadhukhan, S., Hao, Q. and Lin, H. (2017) SIRT7 Is an RNA-Activated Protein Lysine Deacylase. ACS Chem Biol, 12, 300-310.

21. Trott, O. and Olson, A.J. (2010) AutoDock Vina: improving the speed and accuracy of docking with a new scoring function, efficient optimization, and multithreading. $J$ Comput Chem, 31, 455-461.

22. Zhao, X., Allison, D., Condon, B., Zhang, F., Gheyi, T., Zhang, A., Ashok, S., Russell, M., MacEwan, I., Qian, Y. et al. (2013) The 2.5 A crystal structure of the SIRT1 catalytic domain bound to nicotinamide adenine dinucleotide (NAD+) and an indole (EX527 analogue) reveals a novel mechanism of histone deacetylase inhibition. $J$ Med Chem, 56, 963-969.

23. Pan, P.W., Feldman, J.L., Devries, M.K., Dong, A., Edwards, A.M. and Denu, J.M. (2011) Structure and biochemical functions of SIRT6. J Biol Chem, 286, 
$14575-14587$.

24. Priyanka, A., Solanki, V., Parkesh, R. and Thakur, K.G. (2016) Crystal structure of the N-terminal domain of human SIRT7 reveals a three-helical domain architecture. Proteins, 84, 1558-1563.

25. Langelier, M.F., Planck, J.L., Roy, S. and Pascal, J.M. (2012) Structural basis for DNA damage-dependent poly(ADP-ribosyl)ation by human PARP-1. Science, 336, 728-732.

26. Tennen, R.I., Berber, E. and Chua, K.F. (2010) Functional dissection of SIRT6: identification of domains that regulate histone deacetylase activity and chromatin localization. Mech Ageing Dev, 131, 185-192.

27. Wang, D., Kon, N., Lasso, G., Jiang, L., Leng, W., Zhu, W.-G., Qin, J., Honig, B. and $\mathrm{Gu}, \mathrm{W}$. (2016) Acetylation-regulated interaction between p53 and SET reveals a widespread regulatory mode. Nature, 538, 118-122.

28. Hu, S., Pluth, J.M. and Cucinotta, F.A. (2012) Putative binding modes of Ku70-SAP domain with double strand DNA: a molecular modeling study. $J$ Mol Model, 18, 2163-2174.

29. Tang, X., Shi, L., Xie, N., Liu, Z., Qian, M., Meng, F., Xu, Q., Zhou, M., Cao, X., Zhu, W.-G. et al. (2017) SIRT7 antagonizes TGF- $\beta$ signaling and inhibits breast cancer metastasis. Nature Communications, $\mathbf{8}, 318$.

30. Soutoglou, E., Dorn, J.F., Sengupta, K., Jasin, M., Nussenzweig, A., Ried, T., Danuser, G. and Misteli, T. (2007) Positional stability of single double-strand breaks in mammalian cells. Nat. Cell Biol., 9, 675.

31. Tjeertes, J.V., Miller, K.M. and Jackson, S.P. (2009) Screen for DNA-damage-responsive histone modifications identifies H3K9Ac and H3K56Ac in human cells. EMBO J, 28, 1878-1889.

32. Rogakou, E.P., Pilch, D.R., Orr, A.H., Ivanova, V.S. and Bonner, W.M. (1998) DNA double-stranded breaks induce histone H2AX phosphorylation on serine 139. The Journal of biological chemistry, 273, 5858-5868.

33. Paull, T.T. and Lee, J.H. (2005) The Mre11/Rad50/Nbs1 complex and its role as a DNA double-strand break sensor for ATM. Cell Cycle, 4, 737-740.

34. Marechal, A. and Zou, L. (2015) RPA-coated single-stranded DNA as a platform for post-translational modifications in the DNA damage response. Cell Res, 25, 9-23.

35. Ali, A.A.E., Timinszky, G., Arribas-Bosacoma, R., Kozlowski, M., Hassa, P.O., Hassler, M., Ladurner, A.G., Pearl, L.H. and Oliver, A.W. (2012) The zinc-finger domains of PARP1 cooperate to recognize DNA strand breaks. Nat Struct Mol Biol, 19, 685-692.

36. Eustermann, S., Wu, W.F., Langelier, M.F., Yang, J.C., Easton, L.E., Riccio, A.A., Pascal, J.M. and Neuhaus, D. (2015) Structural Basis of Detection and Signaling of DNA Single-Strand Breaks by Human PARP-1. Mol Cell, 60, 742-754.

37. Yang, G., Liu, C., Chen, S.H., Kassab, M.A., Hoff, J.D., Walter, N.G. and Yu, X. (2018) Super-resolution imaging identifies PARP1 and the Ku complex acting as DNA double-strand break sensors. Nucleic Acids Res.

38. Malik, S., Villanova, L., Tanaka, S., Aonuma, M., Roy, N., Berber, E., Pollack, J.R., Michishita-Kioi, E. and Chua, K.F. (2015) SIRT7 inactivation reverses metastatic 
phenotypes in epithelial and mesenchymal tumors. Sci. Rep., 5.

39. Fang, J., Ianni, A., Smolka, C., Vakhrusheva, O., Nolte, H., Kruger, M., Wietelmann, A., Simonet, N.G., Adrian-Segarra, J.M., Vaquero, A. et al. (2017) Sirt7 promotes adipogenesis in the mouse by inhibiting autocatalytic activation of Sirt1. Proc Natl Acad Sci U S A.

40. Tjeertes, J.V., Miller, K.M. and Jackson, S.P. (2009) Screen for DNA - damage responsive histone modifications identifies H3K9Ac and H3K56Ac in human cells. The EMBO journal, 28, 1878-1889.

41. Atsumi, Y., Minakawa, Y., Ono, M., Dobashi, S., Shinohe, K., Shinohara, A., Takeda, S., Takagi, M., Takamatsu, N. and Nakagama, H. ATM and SIRT6/SNF2H Mediate Transient H2AX Stabilization When DSBs Form by Blocking HUWE1 to Allow Efficient gH2AX Foci Formation.

42. Olive, P.L. and Banáth, J.P. (2006) The comet assay: a method to measure DNA damage in individual cells. Nat. Protoc., 1, 23.

43. Falck, J., Coates, J. and Jackson, S.P. (2005) Conserved modes of recruitment of ATM, ATR and DNA-PKcs to sites of DNA damage. Nature, 434, 605. 